\title{
Role of Cardiac CT in the Assessment of Congenital Heart Disease in Children
}

Mohamed Ahmed M. Saad, MD; Abdullah Abbas Abdul Fattah, MD and Sara Ibrahim Abo. Elnour, MD; Alaa Mahmoud Abd El Halim, M.B.B.Ch

Diagnostic Radiology Department, Fayoum University, Fayoum, Egypt

\section{Abstract}

Back Ground: CHD is a common birth defect having significantly increasing. Old assessment for assessment depends on the bair ECHO and angiography. Recent consciences advance in CT.

Aim of Work: To evaluate the value of $\mathrm{CT}$ in assessment of CHD in pediatrics as a non-invasive presurgical planning method and to compare its results with those of ECHO.

Patients and Methods: This research included 50 children with an age up to 12 years-old, evaluated first by ECHO and referred CT examination for further assessment prior surgical interference. Such was conducted in Fayoum University Hospital Radiology Department. The diagnostic susceptibility, relevance, KAPPA and P-values, and ROC was performed according to AUC values of 2 methods.

Results: We compared relevance for ECHO and $\mathrm{CT}$ in the identification of congenital cardiac impairments which chowed superior susceptibility for $\mathrm{ECHO}$ in the detection of heart composition $\mathrm{s}$ and heart vascular communication aberrations, while superior susceptibility for CT for detection of vascular impairments.

Conclusions: The diagnostic susceptibility of both methods for CHD is comparably high with high. Each has its own advantages and disadvantages. ECHO is superior than CT in the identification of atrial septal aberrations, ASD and septal aneurysm. The bair are good in double outlet RV and TGA. On the other hand, CT is sensitive in Aortic pseudocoarctation, Aortic arch aberrations, peripheral PS, and atypical systemic venous drainage and tracing of APCs.

Key words: CT, ECHO, cardiac, complex, CHD and pediatrics.

\section{Introduction}

CHD is the most common of congenital impairment. It was supply thought to such the major cause of mortality neonates was related to the atypical composition and/or function [1].

Cross-sectional imaging became a critical in the diagnostic algorithm in assessment of CHD over the last period. Complimenting ECHO and, superseded the use of diagnostic cardiac catheterization [2].

Although cardiac MR is supply thought to the major imaging modality for CHD, increases in the speed of the $\mathrm{CT}$ and reductions in the radiation hazards rendered CT increasingly used for the assessment of $\mathrm{CHD}$, in particular in neonates and young children [2]. Unlike ECHO, cardiac CT is window independent having wide FOV and provides good assessment of airway composition and coronaries [3].

For the mentioned reasons, cardiac CT is being increasingly utilized in CHD. Recent researches and guideline advices in its use [4].

\section{Patients and methods:}

This research included 50 children up to 12 yearsold from the bair sexes who are known to have complex CHD. CT research was conducted in 
Radiology Department of Fayoum University Hospital.

Inclusion criteria Patients with CHD whom ECHO findings are not sufficient and referred to perform CT for further assessment.

Exclusion criteria Patients who are known to have hyper susceptibility to iodinated $\mathrm{CM}$ reaction, impaired renal functions, respiratory failure, fever and severe asthma and patients with arrhythmia.

Methods All children gave an informed consent prior performing the research. Every child was submitted to full history taking. Clinical assessment of the patients was performed in the form of: measuring of vital signs and anthropometric measurements. ECHO was performed by Vivid S5 GE Ultrasound Machine. Patients were prepared for $\mathrm{CT}$ as following: calculation of amount of $\mathrm{CM}$ and sedations. The patient were fasting for 4 hours. The peripheral venous line was placed usually in a right upper limb vein, exercise if cosurgical. The patients were put in a supine position and at the middle of CT gantry. ECG leads were put on the chest of the patient. Infants below 6 months were lying with arms at their side or above their head for image acquisition, positioning the arms above their head is advice ined, while patients above 6 months of age were positioned with their arms above their head when possible. CT examination was done by 160 MDCT Toshiba Machine:

- We used the following parameters during cardiac CT scanning:

- Pitch of 1.3, helical thickness of $0.5 \mathrm{~mm}$ and coverage of $32 \mathrm{~mm}$.

- The radiation dose set to 80-100 kvp.

- Tube current from 10 to $40 \mathrm{~mA} / \mathrm{kg}$.

- The gantry speed at a 0.35 -s rotation.

- Prospective gating.

- Wide FOV.
- A scout was taken and dual-phase injection conducted using Omnipaque $350 \mathrm{mg} / \mathrm{mL}$ injection. The total contrast volume was $1.5-3$ $\mathrm{ml} / \mathrm{kg}$.

- Scanning begins when contrast filled the LV by bolus tracking.

- All images were transferred to workstation for reporting,

\section{Statistical analysis of information}

Information were collected and coded to facilitate information manipulation and double entered into Microsoft Access and information analysis was performed using the Statistical Package of Social Science software version 22 in windows 7. Simple descriptive analysis in the form of numbers and percentages of qualitative data, arithmetic means as central tendency measurement and standard deviations as measure of dispersion.

For quantitative information Independent samples $\mathrm{t}$ test.

\section{For qualitative information}

- Chi-square test, KAPPA value and P-value.

- Susceptibility, relevance and diagnostic area under the ROC curve according to AUC value of 2 .

\section{Ethical Considerations}

This research was reviewed by the Fayoum University's Faculty of Medicine Research Ethical Committee.

\section{Results}

Baseline Demographic Characteristics of Children with CHD in the Research We recruited 50 children in Radiology Department-FUH, from October 2019 to April 2020. Aged up to 12 years- 
ISSN: 2536-9482 (Online) Fayoum University Medical Journal Saad et al., 2021,1(1), 1-11

old from both sexes. $60 \%$ of patients were females

and $40 \%$ were males, as also revealed in (Fig. 1).

Fig. 1: Chart illustrating the frequency of females and males among research group

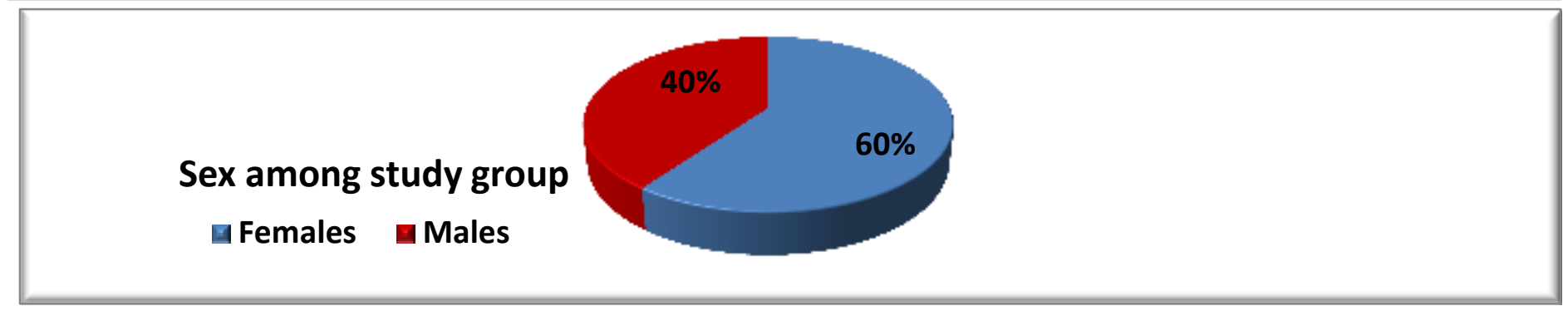

(Fig. 2) demonstrated 58\% suffered chest symptoms, $42 \%$ had cyanosis and $42 \%$ had delayed milestones.

Fig. 2: Chart illustrating the frequency of non-identical symptoms among research group.

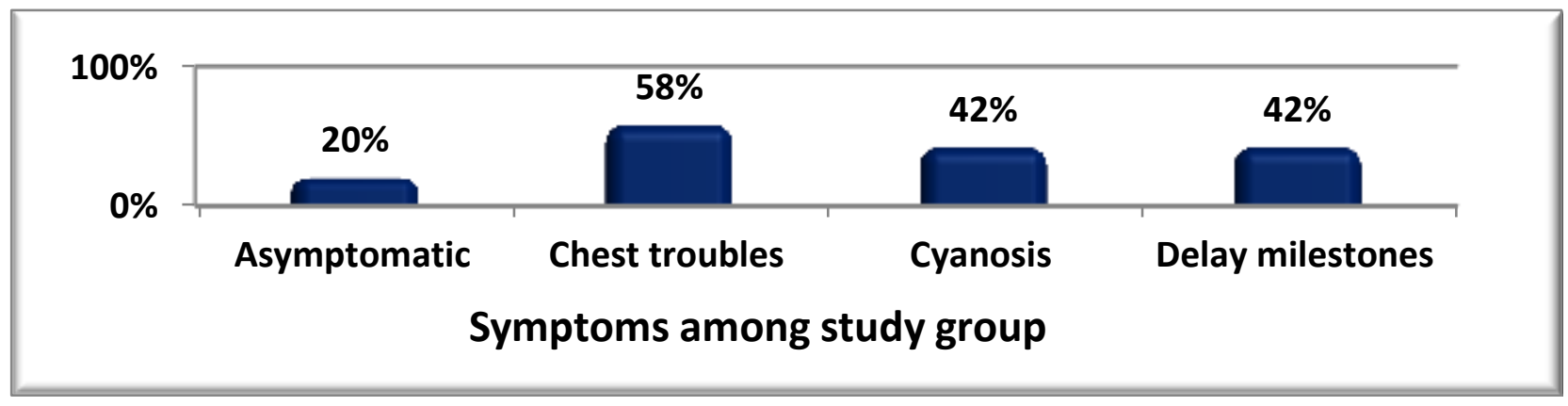

Sedation in CT As in table (2) and (Fig. 3)., we needed to supply sedation in 34 patients reciprocal to $68 \%$ of the research subjects versus $32 \%$ of patients, who performed the research without sedation.

Table 2: Frequency of sedation use in CT among research group.

\begin{tabular}{|c|c|c|}
\hline \multirow{2}{*}{$\begin{array}{c}\text { Variables } \\
(\mathbf{n = 5 0})\end{array}$} & \multicolumn{2}{|c|}{ Need for CT sedation } \\
\cline { 2 - 3 } & Number & $\%$ \\
\hline No & 16 & $32 \%$ \\
Yes & 34 & $68 \%$ \\
\hline
\end{tabular}

Fig. 3: Illustrative chart for sedation use in CT among research group.

Need for MDCT sedation 国 No sedation

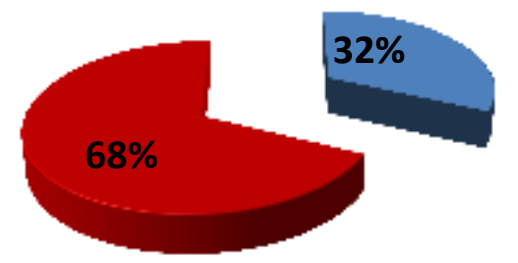


Fayoum University Medical Journal Saad et al., 2021,1(1), 1-11

Table 3: The diagnostic analysis of $\mathrm{CHD}$ by ECHO among research group.

\begin{tabular}{|c|c|c|c|c|}
\hline \multirow[b]{2}{*}{ CHD evaluated by ECHO } & \multicolumn{4}{|c|}{ Number of patient findings $(n=50)$} \\
\hline & $\begin{array}{c}\text { Anomaly } \\
\text { does not } \\
\text { exist }\end{array}$ & $\begin{array}{l}\text { Mis- } \\
\text { recognized }\end{array}$ & $\begin{array}{c}\text { Under } \\
\text { recognized }\end{array}$ & Discovered \\
\hline \multicolumn{5}{|l|}{ Heart composition s impairments: } \\
\hline ASD & 28 & 0 & 0 & 22 \\
\hline PFO & 38 & 0 & 1 & 11 \\
\hline SEPTAL ANEURYSM & 47 & 0 & 0 & 3 \\
\hline VSD & 19 & 0 & 0 & 31 \\
\hline Single atrium & 48 & 0 & 0 & 2 \\
\hline Single ventricle & 45 & 0 & 0 & 5 \\
\hline RVOTO & 33 & 0 & 0 & 17 \\
\hline TV impairments & 44 & 0 & 1 & 5 \\
\hline MV impairments & 44 & 0 & 0 & 6 \\
\hline \multicolumn{5}{|c|}{ Heart-vascular communication impairments: } \\
\hline DOULKBLE OULTLET RV & 42 & 0 & 0 & 8 \\
\hline PV impairments & 16 & 0 & 0 & 34 \\
\hline Aortic valve impairments & 46 & 1 & 0 & 3 \\
\hline TGA & 43 & 0 & 0 & 7 \\
\hline \multicolumn{5}{|l|}{ Vascular impairments: } \\
\hline Aortic pseudocoarctation & 47 & 2 & 1 & 0 \\
\hline Aortic coarctation & 42 & 0 & 4 & 4 \\
\hline Aortic arch abberations & 41 & 8 & 0 & 1 \\
\hline Pulmonary artery dilatation & 31 & 0 & 2 & 17 \\
\hline Peripheral PS & 30 & 6 & 5 & 9 \\
\hline PDA & 26 & 1 & 3 & 20 \\
\hline Atypical pulmonary venous drainage & 45 & 1 & 1 & 3 \\
\hline Atypical systemic venous drainage & 40 & 6 & 0 & 4 \\
\hline Coronary arteries abberations & 46 & 3 & 1 & 0 \\
\hline APCs & 36 & 5 & 4 & 5 \\
\hline \multicolumn{5}{|l|}{ Total (No., \%): } \\
\hline \multirow[b]{2}{*}{23 types of CHD } & \multirow[b]{2}{*}{---} & $33(12.09 \%)$ & $23(8.43 \%)$ & $217(79.48 \%)$ \\
\hline & & \multicolumn{3}{|c|}{$\begin{array}{c}\text { Total encountered } \\
\text { abberations }=273(100 \%) \\
\end{array}$} \\
\hline
\end{tabular}


Fayoum University Medical Journal Saad et al., 2021,1(1), 1-11

Table 4: The diagnostic analysis of CHD by CT among research group.

\begin{tabular}{|c|c|c|c|c|}
\hline \multirow[b]{2}{*}{ CHD evaluated by CT } & \multicolumn{4}{|c|}{ Number of patient findings $(n=50)$} \\
\hline & $\begin{array}{c}\text { Anomaly } \\
\text { does not } \\
\text { exist }\end{array}$ & $\begin{array}{c}\text { Mis- } \\
\text { recognized }\end{array}$ & $\begin{array}{l}\text { Under } \\
\text { recognized }\end{array}$ & Discovery \\
\hline \multicolumn{5}{|l|}{ Heart composition s impairments: } \\
\hline ASD & 28 & 3 & 3 & 16 \\
\hline $\mathrm{PFO}$ & 38 & 6 & 3 & 3 \\
\hline SEPTAL ANEURYSM & 47 & 0 & 3 & 0 \\
\hline VSD & 19 & 0 & 0 & 31 \\
\hline Single atrium & 48 & 0 & 0 & 2 \\
\hline Single ventricle & 45 & 0 & 2 & 3 \\
\hline RVOTO & 33 & 3 & 0 & 14 \\
\hline TV impairments & 44 & 2 & 2 & 2 \\
\hline MV impairments & 44 & 2 & 1 & 3 \\
\hline \multicolumn{5}{|c|}{ Heart-vascular communication impairments: } \\
\hline DOULKBLE OULTLET RV & 42 & 0 & 2 & 6 \\
\hline PV impairments & 16 & 2 & 2 & 30 \\
\hline Aortic valve impairments & 46 & 1 & 0 & 3 \\
\hline TGA & 43 & 0 & 1 & 6 \\
\hline \multicolumn{5}{|l|}{ Vascular impairments: } \\
\hline Aortic pseudocoarctation & 47 & 0 & 0 & 3 \\
\hline Aortic coarctation & 42 & 0 & 1 & 7 \\
\hline Aortic arch abberations & 41 & 0 & 0 & 9 \\
\hline PA dilatation & 32 & 0 & 0 & 18 \\
\hline Peripheral PS & 30 & 0 & 0 & 20 \\
\hline PDA & 26 & 3 & 3 & 18 \\
\hline $\begin{array}{l}\text { Atypical pulmonary venous } \\
\text { drainage }\end{array}$ & 45 & 0 & 1 & 4 \\
\hline Atypical systemic venous drainage & 40 & 0 & 0 & 10 \\
\hline Coronary arteries abberations & 46 & 0 & 0 & 4 \\
\hline APCs & 36 & 0 & 0 & 14 \\
\hline \multicolumn{5}{|l|}{ Total (No., \%): } \\
\hline \multirow[b]{2}{*}{23 types of CHD } & \multirow[b]{2}{*}{---} & $22(8.06 \%)$ & $24(8.79 \%)$ & $227(83.15 \%)$ \\
\hline & & \multicolumn{3}{|c|}{$\begin{array}{c}\text { Total encountered } \\
\text { abberations }=273(100 \%)\end{array}$} \\
\hline
\end{tabular}




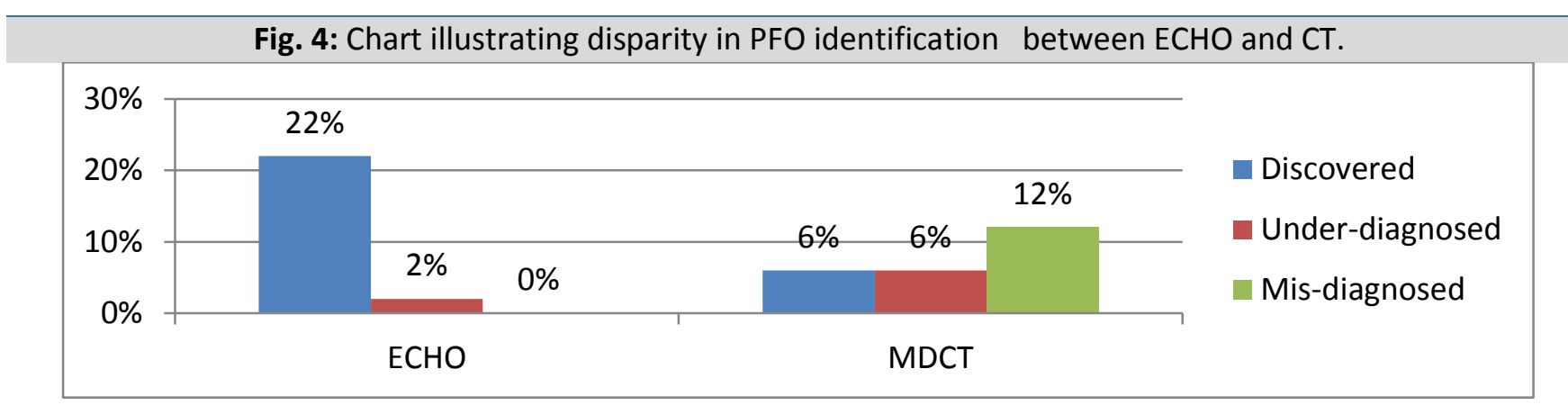

Fig. 5: Chart illustrating disparity in the identification of Aortic pseudocoarctation between ECHO and CT.
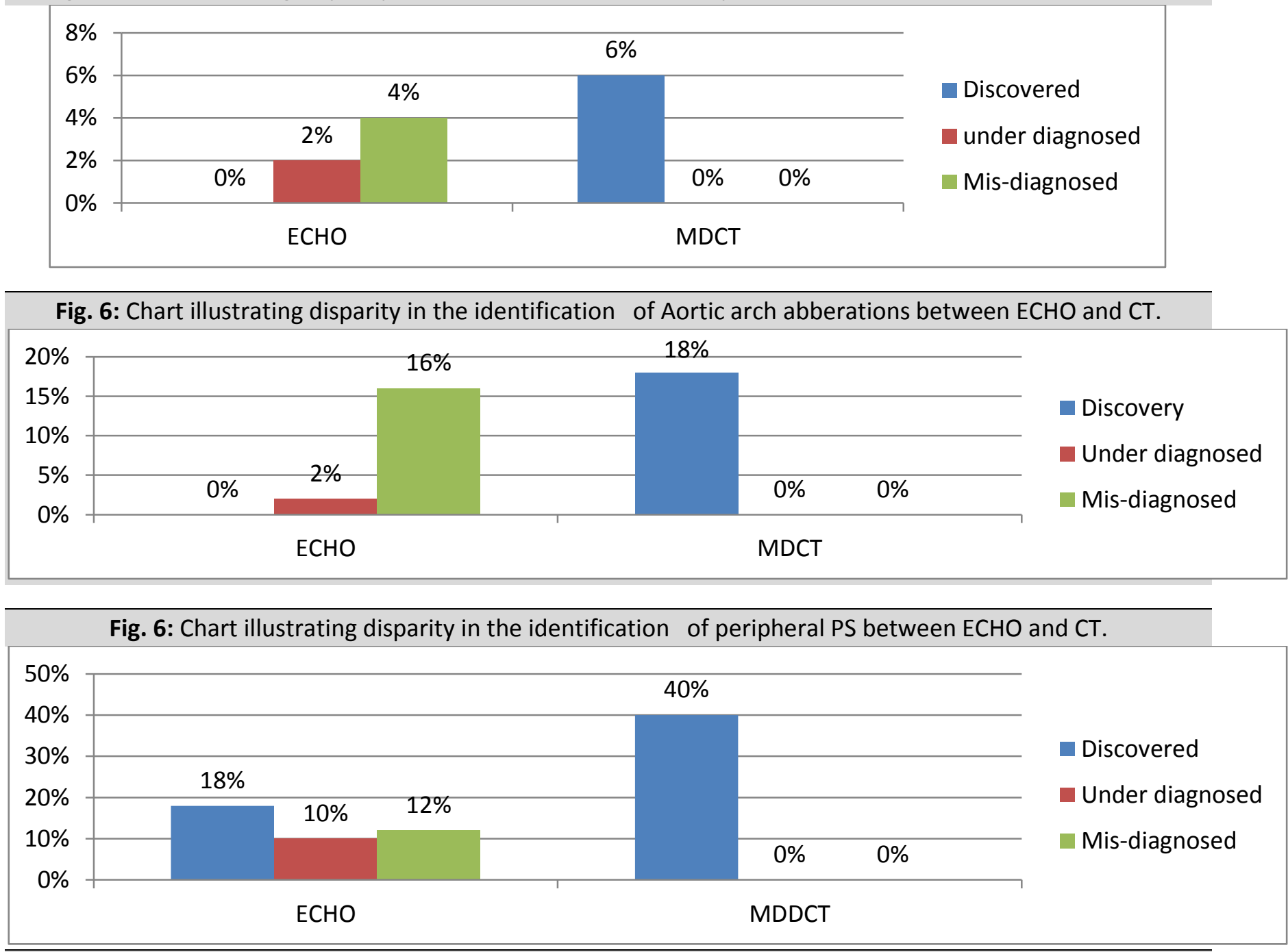

Fig. 7: Chart illustrating disparity in the identification of atypical systemic venous drainage between $\mathrm{ECHO}$ and CT. 


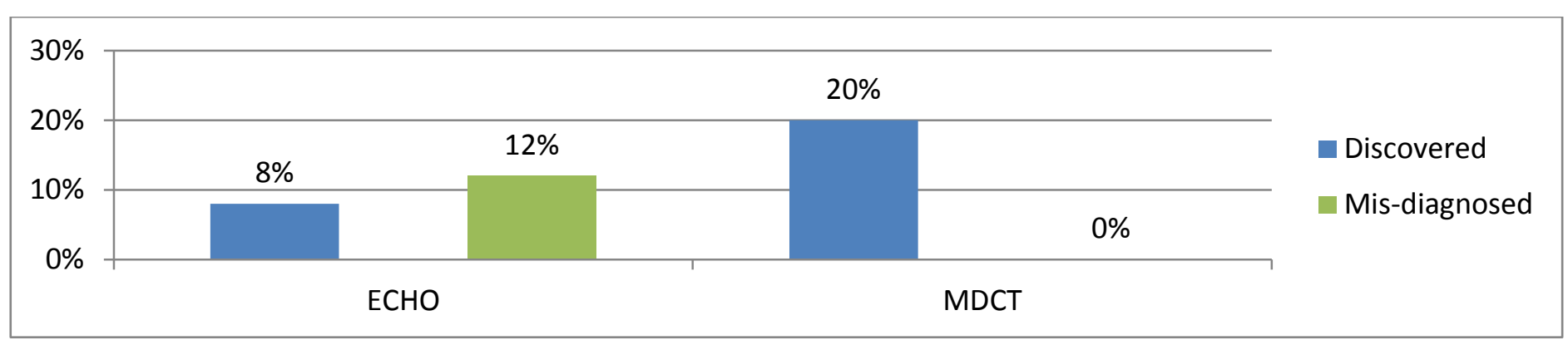

Fig. 8: Chart illustrating disparity in the identification of coronary arteries abberations between ECHO and CT.
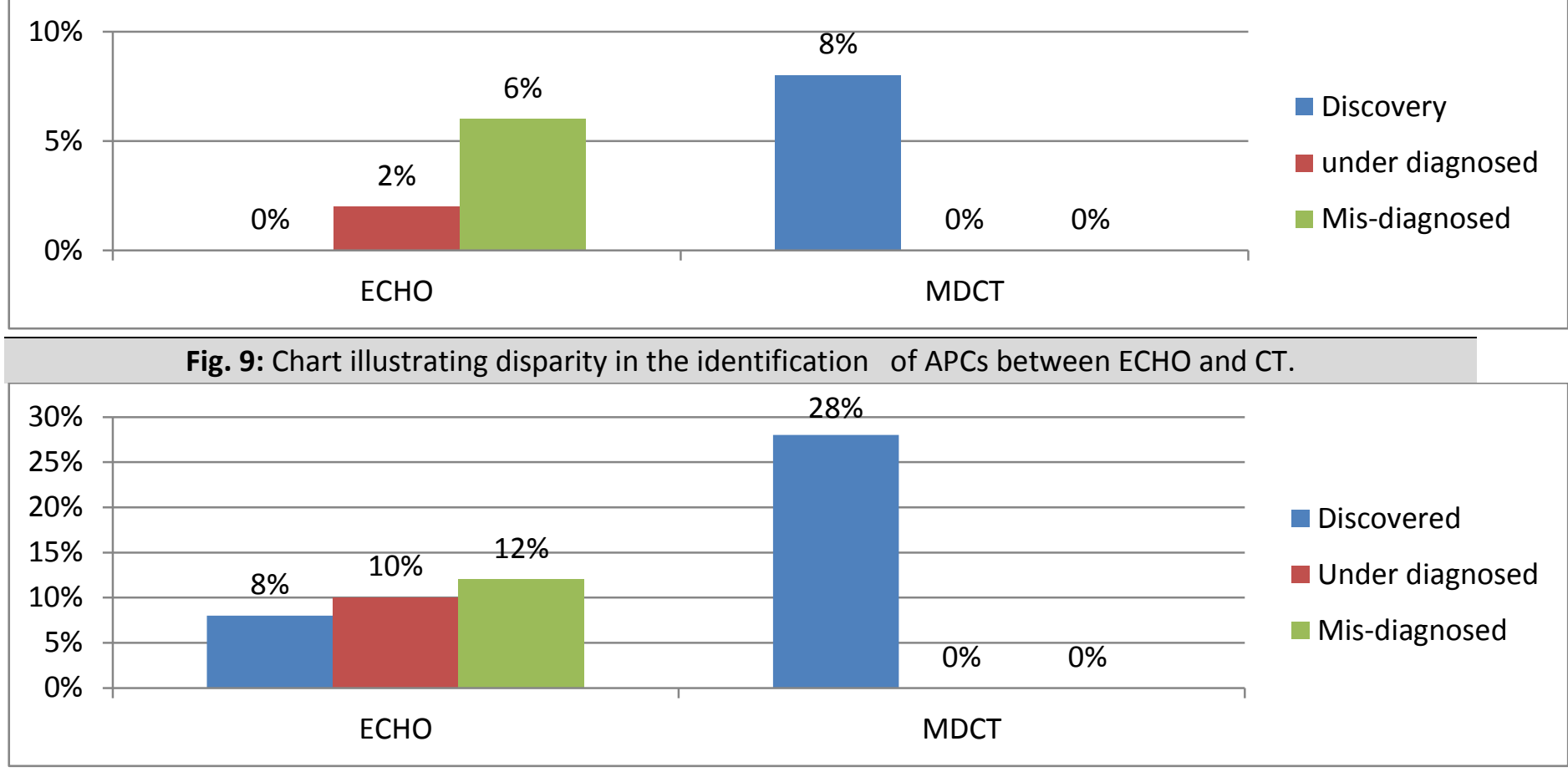

Comparison between ECHO and CT in Mc-goon's ratio

Table 5: Comparison between ECHO and CT in Mc-goon's ratio. *: A statistically significant value.

\begin{tabular}{|c|c|c|c|}
\hline \multirow{2}{*}{ Variables } & \multicolumn{2}{|c|}{ Mc-goon's ratio } & \multirow{2}{*}{$\boldsymbol{P}$-value } \\
\cline { 2 - 3 } & Mean & SD & \multirow{2}{*}{$<0.001 *$} \\
\hline ECHO & 1.69 & 0.49 & $<0.65$ \\
\hline
\end{tabular}

Table 162: The mean, SD and range of Mc-goon's ratio measured by ECHO and CT

\begin{tabular}{|c|c|c|c|}
\hline Variables & Mean & SD & Range \\
\hline \multicolumn{4}{|c|}{ Mc-goon's ratio (n=22) } \\
\hline ECHO & 1.69 & 0.49 & $1.1-3$ \\
\hline MDSCT & 1.99 & 0.65 & $1-4$ \\
\hline
\end{tabular}


Fig. 13: Illustrative chart for Mc-goon's ratio mean in the research.

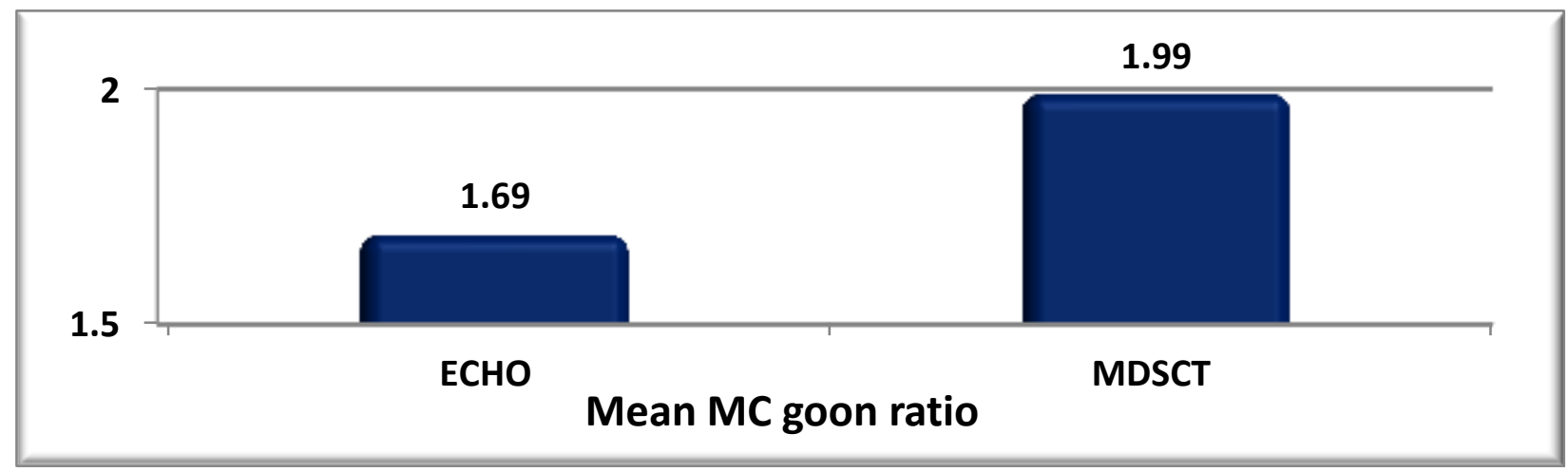

\section{Discussion}

\begin{abstract}
CHD is thought
to be common accounting for around $50 \%$ of the patients. Accurate presurgical assessment of complex CHD is critical for selection of the good surgical approach [5]. Advances in medical and surgical care of patients with CHD have resulted in expected survival for even the most complex afflictions [6]. ECHO is used to be the first line imaging modality for identification and follow up of neonates with CHD, minimizing the need for diagnostic cardiac catheterization [7].
\end{abstract}

In the last two decades, CT scan emerged as a worthy non-invasive cardiovascular diagnostic tool efficient in providing accurate anatomic information not obtainable by other imaging modalities [8]. The improved spatial and temporal resolution, rapid image acquisition and radiation dose reduction of newer generation scanners have dramatically increased the applicability of CT scanning to patients with CHD [9]. CT is used in patients with CHD in patient when ECHO is not sufficient and MRI [10].

CT optimally used in limited settings, where all advanced diagnostic tests are available, so such the modality with the best information and least risk [11]. CT has the criteria of fast scanning speed, high temporal/spatial resolution, good post-processing for images and =visualization of the anatomic structures [12].

Among the 50 children in the research, we encountered 273 aberrations in total, which further specified into 23 non identical types of CHD. These 23 types of CHD were grouped under 3 major headlines: heart compositions, heart-vascular communication and vascular impairments. ECHO findings were classified into: discovered, under recognized, misrecognized and/or non-existent aberrations. Among research group, ECHO discovered 217 aberrations, reciprocal to $79.48 \%$ of total encountered aberrations in the research. The largest number of discovered heart compositions impairments by ECHO was for VSD of 31 patients. For heart-vascular communication impairments, the largest number of discovered aberrations was for PV impairments of 34 patients. Among vascular impairments, the largest number of discovered aberrations was for PDA of 20 patients all around the research. ECHO under recognized 23 aberrations, reciprocal to $8.43 \%$ of total encountered aberrations in the research, meaning such we needed further confirmation of findings, proper reassessment of the anatomic structural details and/or measurements by the CT as a pre- 
interventional step, and then we found insufficient information obtained by ECHO compared to those of $\mathrm{CT}$ in the assessment of these existent aberrations. The most under recognized anomaly by ECHO such needed CT assessment was the peripheral PS accounting for 5 patients all around the research. ECHO miss recognized 33 aberrations, reciprocal to $12.09 \%$ of total encountered aberrations in the research, which were then discovered by CT. The most mis-recognized aberrations by ECHO were the Aortic arch aberrations of 8 patients all around the research. Table (3) demonstrated the number of patients for each type of CHD grouped into: heart compositions, heart-vascular communication and vascular impairments evaluated by ECHO in details.

Furthermore, we assessed the encountered 273 aberrations from non-identical types of CHD by CT. Table (4) demonstrated, in details, the number of patients for each CHD type evaluated by CT. CT discovered 227 aberrations, which correspond to $83.15 \%$ of the total encountered aberrations in the research. The largest number of discovered heart compositions impairments by CT was also for VSD of 31 patients, similar to ECHO findings. For heart-vascular communication impairments, the largest number of discovered aberrations was as ECHO for PV impairments with a close number of patients to ECHO of 30 patients. Among vascular impairments, CT results differed from such those of ECHO, as the largest number of discovered aberrations was for peripheral PS of 20 patients all around the research. CT under recognized 24 aberrations, which correspond to $8.79 \%$ of the total encountered aberrations in the research, meaning such, we needed further confirmation by other diagnostic modalities, and then we found insufficient information abstained by CT. The most under-recognized aberrations by $\mathrm{CT}$ were atrial septal aberrations and PDA from vascular impairments of 3 patients for each all around the research. CT miss recognized 22 aberrations discovered by ECHO and/or other modalities, which correspond to $8.06 \%$ of the total encountered aberrations in the research. The largest number of mis-recognized aberrations was for PFO of 6 patients. The frequency of CHD discoveries was superior in CT than ECHO accounting to $83.15 \%$ and $79.48 \%$ of encountered aberrations in the research, respectively. On the other hand, the frequency of CHD under recognizes was slightly superior in $\mathrm{CT}$ than ECHO accounting to $8.79 \%$ and $8.43 \%$ of encountered aberrations in the research, respectively. The frequency of mis-recognizes was much superior in ECHO than CT accounting to $12.09 \%$ and $8.06 \%$ of total encountered aberrations in the research, respectively.

Regarding the heart compositions impairments with a significant statistical disparity between ECHO and CT in in this research with P-value < 0.05 , we found such $24 \%$ of patients in this research had PFO in association with other aberrations. Only $2 \%$ were under recognized by $\mathrm{ECHO}$, due to insignificant results as disparity's in size, while $12 \%$ were mis-recognized and did not visualized by CT, as revealed in (Fig. 4).

Regarding each type of vascular impairments, which revealed a significant statistical disparity between ECHO and CT in this research with Pvalue $<0.05$, we found such $18 \%$ of patients had Aortic pseudocoarctation, proved by the kinking appearance of the Aorta with no collaterals detected in CT. All of them were discovered by CT, while errors occurred by ECHO, as $4 \%$ of patients were falsely recognized as Aortic coarctation, while $2 \%$ of patients were have misgivings about and referred to CT for further assessment, as revealed in (Fig. 5). 
$18 \%$ of patients in this research had non identical types of Aortic arch aberrations, including: double aortic arch, right-sided Aortic arch, aberrant right SCA, atypical vertebral artery origin and Arch hypoplasia. Also, all of them were discovered by $\mathrm{CT}$, while ECHO miss recognized $16 \%$, as revealed in (Fig. 5).

$40 \%$ of patients in the research had peripheral PS at non identical levels. All of these aberrations were discovered by CT. No CT peripheral PS under or mis-recognizes. By ECHO, only $18 \%$ were discovered, while $10 \%$ were have misgivings about and $12 \%$ were miss recognized, as revealed in (Fig. 6).

$20 \%$ of patients, had non identical types of atypical systemic venous drainage, including: PERSESTANT LEFT SVC, interrupted IVC, double IVC and retro-Aortic innominate vein. All were discovered by CT. By ECHO, only $8 \%$ were discovered, the largest percentage as for miss recognized as in (Fig. 7).

There were $8 \%$ of patients suffered ho had coronary arteries aberrations, including: coronary artery fistulae, aneurysms and atypical coronaries origin. All were discovered by CT, while errors occurred by ECHO. $6 \%$ of patients miss recognized by ECHO and $2 \%$ of patients were have misgivings about, as revealed in (Fig. 8).

We found $28 \%$ of patients had APCs, otally discovered by CT. By ECHO, only $8 \%$ were discovered, $10 \%$ were under recognized as we needed further assessment of APCs with significant non identical information gained by CT in these patients. $10 \%$ of patients were miss recognized by ECHO, as revealed in (Fig. 9).

Mc-goon ratio was calculated by dividing the sum of the cranio-caudal diameter of the right PA at the pre-branching and that of the left PA by the Aortic diameter at the level of diaphragm. It is considered normal when ranging from 2 to 2.5 . Table (5) demonstrated such there was a significant statistical disparity with $\mathrm{P}$-value < 0.05 between ECHO and CT in measurement of Mc-goon's ratio with slightly superior mean among CT. Table (6) demonstrated such the mean Mc-goon's ratio by ECHO was $1.69 \pm 0.49$ with a range from 1.1 to 3 , but when measured by $\mathrm{CT}$, the mean was $1.99 \pm 0.65$ with a range from 1 to 4 , as revealed in (Fig. 13).

\section{Conclusion}

We advise in ECHO in first line identification establishment, rechecking and following up the afflictions, for most of the heart structures and impairments. We advise in CT in for macrovascular impairments, tracing of APCs and coronaries i.e. MDCT for detailed vascular components and ECHO for gross cardiac components.

\section{References}

1 Hou Q-R, Gao W, Sun A-M, Wang Q, et al. A prospective assessment of contrast and radiation dose and image quality in cardiac $\mathrm{CT}$ in children with complex congenital heart disease using low-concentration iodinated contrast agent and low tube voltage and current. Br J Radiol 2017; 90:1.

2 Gianfranco Butera, Massimo Chessa, Andreas Eicken and John D. Atlas of Cardiac Catheterization for Congenital Heart Disease published by the company Springer Switzerland AG. Radiographics. 2019;40:351.

3 Joseph Schoepf, Anthony M. and Hlavacek. CT of the Heart.Originally, Contemporary Cardiology by the registered company Springer Nature Switzerland. Thorac Cardiovasc Surg. 2019;20:44(2):227-559. 
4 Cohen MS, Eidem BW, Cetta F, Fogel MA, et al. Multimodality imaging guidelines of patients with transposition of the great arteries: a report from the American Society of Echocardiography developed in collaboration with the Society for Cardiovascular Magnetic Resonance and the Society of Cardiovascular Computed Tomography. J Am SocEchocardiogr.2016;29(7):571-621.

$5 \mathrm{Hu}$ L., Gao W., Zhong Y., Sun A., et al. Diagnostic accuracy of sub-mSv prospective ECGtriggering cardiac CT in young infant with complex congenital heart disease. The International Journal of Cardiovascular Imaging, June 2008;(32):991-998.

6 Marelli AJ, Ionescu-Ittu R, Mackie AS, Guo L, et al. Lifetime prevalence of congenital heart disease in the general population from 2000 to 2010. Circulation. 2014;130(9):749-56.

7 Lai W., Forbus, G.A. and Shirali, G.S. Abberations of the Ventricular Septum. Echocardiography in Pediatric and Congenital Heart Disease: From Fetus to Adult. Blackwell Publishing Ltd. 2008(1):176-187.

8 Corno F.A. Congenital heart defects decision for Cardiac surgery, Pediatr Cardiol. August 2008(3),95.

9 Han BK, Lesser AM, Vezmar M, Rosenthal K, et al. Cardiovascular imaging trends in congenital heart disease: a single center experience. J Cardiovasc Comput Tomogr. 2013;7(6):361-6

10 Han BK, Rigsby CK, Leipsic J, Bardo D, et al. Computed tomography imaging in patients with congenital heart disease, part 2: technical advice inations. An expert consensus document of the Society of Cardiovascular Computed Tomography (SCCT): The Society of Pediatric Radiology (SPR) and the North American
Society of Cardiac Imaging (NASCI). J Cardiovasc Comput Tomogr. 2015;9(6):493513.

11 Taylor AJ, Cerqueira M, Hodgson JM, Mark D, et al. Appropriate use criteria for cardiac computed tomography. A report of the American College of Cardiology foundation appropriate use criteria task force, the North American Society for Cardiovascular Imaging, the Society for Cardiovascular Angiography, the Society for Cardiovascular Magnetic Resonance. J Cardiovasc Comput Tomogr. 2010;4(6):407 e133.

$12 \mathrm{Bu}$ G., Miao Y., Bin J., Deng S., et al. Comparison of 128-Slice Low-Dose Prospective ECG-Gated CT and Trans-Thoracic Echocardiography for Congenital Heart Disease, Journal Rad 2016;11(10): 40-29.

13 Dillman J.R., Yarram S.G. and Hernandez R.J. Imaging of pulmonary venous developmental aberrations. AJR Am J Roentgenol 2009; 192 (5): 1272-1285 